Vol 11, Issue 7, 2018

\title{
IN SILICO AND IN VIVO PHARMACOLOGICAL STUDIES OF CLOZAPINE AND D-AMINO ACID OXIDASE INHIBITOR FOR COGNITIVE ENHANCEMENT
}

\author{
NITHYA $S^{1}$, SHANMUGARAJAN TS ${ }^{2 *}$ \\ ${ }^{1}$ Department of Pharmacology, School of Pharmaceutical Sciences, Vels Institute of Science Technology and Advanced Studies, Pallavaram, \\ Chennai - 600 117, Tamil Nadu, India. ${ }^{2}$ Department of Pharmaceutics, School of Pharmaceutical Sciences, Vels Institute of Science \\ Technology and Advanced Studies, Pallavaram, Chennai - 600 117, Tamil Nadu, India. Email: smrajan@velsuniv.ac.in
}

Received: 19 April 2018, Revised and Accepted: 19 May 2018

ABSTRACT

Objective: D-amino acid oxidase inhibitors (DAAOIs) are of particular focus for cognition study. Atypical antipsychotics are known DAAO inhibitors. The present examination was done to check out the binding affinity of atypical antipsychotics by docking toward the DAAO protein; in conclusion, the picked antipsychotic drug was checked for their cognition enhancing activity with scopolamine-induced amnesia.

Methods: The crystal structure of DAAO was obtained from Protein Data Bank, the energy minimization was performed with CHARMM program, then active site prediction was made out using Ramachandran plot, and finally, docking examination was finished using Autodock 4.2 tool. For in vivo study, the mice were divided into three groups. Group I - vehicle (Saline) treated, Group II - saline +scopolamine (1 mg/kg, intraperitoneal [i.p]) treated, and Group III - clozapine (20 mg/kg, i.p) + scopolamine (1 mg/kg, i.p).

Results: The Autodock examination shows significant binding affinity of -5.22 for brexpiprazole and least or positive binding affinity of +1 for iloperidone. Clozapine with binding energy of -2.87 was decided for completing the in vivo cognition study. The in vivo shows up that clozapine $(20 \mathrm{mg} / \mathrm{kg}$, i.p) exhibits a change in the impairment of spatial memory.

Conclusion: The results recommend that the clozapine produces cognitive enhancement through both DAAOI and antipsychotic action. Clozapine has cognitive improvement potential, favoring its usage in reducing toxic impacts of scopolamine

Keywords: D-amino acid oxidase, Antipsychotics, Clozapine, Cognition.

(C) 2018 The Authors. Published by Innovare Academic Sciences Pvt Ltd. This is an open access article under the CC BY license (http://creativecommons. org/licenses/by/4. 0/) DOI: http://dx.doi.org/10.22159/ajpcr.2018.v11i7.26800

\section{INTRODUCTION}

D-amino acid oxidase (DAAO) is a flavoenzyme that degrades the D-amino acids through the system of oxidative deamination. DAAO control of keeping up D-amino acid levels has been connected with a couple of physiological techniques running from hormone secretion to synaptic transmission and cognition [1]. Preclinical figures suggest that second-generation antipsychotics could diminish cognitive impairment [2]. Second-generation antipsychotics have regularly outflanked their original partners in switching or weakening phencyclidine- or methamphetamine-instigated shortfalls in inversion learning, restrictive learning, consideration, spatial memory, spatial learning, and long-haul potentiating effect in rodents and nonhuman primates [3].

In this work, we predict the three-dimensional (3D) structure and the protein-ligand and protein-protein docking of DAAO using various Bioinformatics methods. The essential purpose of our investigation was to expect the 3D structure and docking. The objective of the present examination was to clear up the participations of DAAO proteins with ligands and distinctive proteins and to perceive the relationship of DAAO to insight. Protein-protein docking and association reproduction reveal hydrogen and ionic bonds.

Scopolamine, a non-selective muscarinic receptor antagonist, causes debilitation in learning and memory by decreasing cholinergic action. On intraperitoneal (i.p.) administration of scopolamine, the cholinergic neurotransmission was blocked, provoking cholinergic brokenness and obstructed cognitive impairment in mice [4]. Starting late, it has been represented that memory impairment induced by scopolamine in mice is connected with changed cerebral amino acid levels by the protein
DAAO. Along these lines, mice with scopolamine-induced memory deficiencies were used as an animal model for the screening of cognitive enhancement agents.

Here, we investigate whether the neuroprotective effect of clozapine reduced learning and memory impairment induced by a muscarinic antagonist scopolamine in mice. We assessed the effect of clozapine on scopolamine-induced learning and memory impairment with Morris water maze tests.

\section{MATERIALS AND METHODS}

\section{In silico method}

Retrieval of crystal structure and target preparation

The high-resolution crystal structure of human DAAO was retrieved from the Protein Data Bank. FASTA sequence of the retrieved structure revealed the presence of glycine at position. The target was preprocessed by standard methods before binding analysis [5].

\section{Energy minimization [6]}

CHARMM is a general and flexible program for macromolecular energy minimization and dynamic calculations that utilize both classical and quantum mechanical energy functions for molecular systems. Energy minimization of target enzyme was carried out under CHARMM27 force field. Gradient was set to 0.05 .

\section{Structural assessment of proteins [7]}

3D structure and conformational stability of the protein were analyzed by means of Ramachandran plot. 
Active site prediction [8]

The ligand-binding domain of the human DAAO was predicted using the Site Finder module of the molecular operating environment.

\section{Molecular docking analysis [9]}

To understand the binding conformation and affinity, antipsychotics were individually docked to the active site of human DAAO. Autodock 4.2 tool was used for molecular docking analysis. Both the receptor and ligands were prepared by the addition of hydrogen and Gasteiger charges. A grid defining the active site was constructed before running the docking simulation. Genetic algorithm was adopted for conformer search while docking.

\section{In vivo methods}

Animals

Male Swiss Albino mice (25-30 g: 24 mice) were obtained from the Animal house, TANUVAS, Chennai, and housed into four groups of six mice each. The mice were housed in cages at a distorted temperature of $25^{\circ} \mathrm{C} \pm 2^{\circ} \mathrm{C}$ with a $12 \mathrm{~h} \mathrm{light/dark} \mathrm{cycle.} \mathrm{The} \mathrm{animals} \mathrm{had} \mathrm{free} \mathrm{access} \mathrm{to} \mathrm{standard} \mathrm{pellet} \mathrm{eating}$ regimen and drinking water. Behavioral studies were performed in a silent room between 9.00 am and 11.00 am to stay away from circadian variation. The investigation was endorsed by the Institutional Animal Ethical Committee, and work was completed according to the CPCSEA Guidelines, New Delhi. XIX/VELS/PCOL/05/2000/CPCSEA/IAEC/03.10.2016

\section{Drugs and chemicals}

Scopolamine (Sigma-Aldrich), Clomach (La Pharmaceuticals, Ahmedabad), and sodium chloride (Sigma-Aldrich) were utilized. Every other substance and reagents used are of analytical grade.

\section{Experimental groups}

- Group I - Saline treated (10 mL/Kg, i.p.,) (Vehicle)

- Group II - Saline (10 mL/Kg, i.p.,)+scopolamine (1 mg/Kg, i.p) treated,

- Group III - Clozapine (20 mg/Kg, i.p)+scopolamine (1 mg/Kg, i.p).

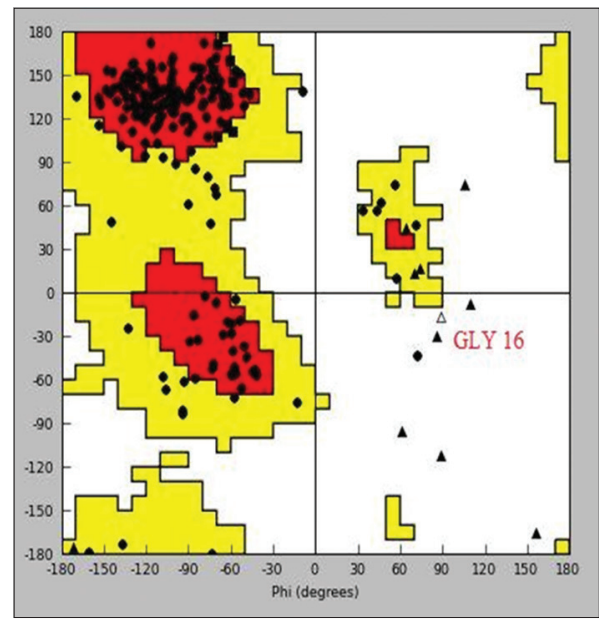

Fig. 1: Ramachandran plot of D-amino acid oxidase

\section{Treatment schedule [10]}

To observe the impact of clozapine, antipsychotic drug on scopolamine induced amnesia in eighteen mice (6 in every group were divided into three groups), the control group received vehicle (saline, $0.9 \% \mathrm{NaCl}$ 2) once daily for 18 days, the second group received saline for 15 days followed by scopolamine ( $1 \mathrm{mg} / \mathrm{kg}$ i.p.) for 3 days, and the third group received Clozapine $(20 \mathrm{mg} / \mathrm{kg}$, i.p) for 15 days followed by scopolamine (1 mg/kg i.p.) for 3 days All drugs were ready, freshly prepared and given once daily within the morning and followed an equivalent program. The training and memory parameters were performed 120 min once after drug administration. On the day 18, behavioral study for finding the escape latency and time spent on platform quadrant are carried out.

\section{Learning and memory evaluation: Morris maze test [11]}

Spatial learning and memory were assessed by the Morris water maze. The technique included two stages. The initial step was the place navigation test from day 1 to 4 , in which the escape latency (EL) (the time required to escape onto the hidden platform) was utilized to assess learning and memory function. Mice that found the platform were permitted to stay on the platform for $20 \mathrm{~s}$ and were then come back to the home cage. On the off chance that mice did not achieve the platform inside $120 \mathrm{~s}$, it was tenderly guided to the platform by the experimenter, where it stayed for $20 \mathrm{~s}$. The last trial was viewed as the probe test. The second step was the spatial test on day 5 after evacuation of the platform and after the probe test, which was performed to test the capacity of mice to locate the expelled platform by memory

\section{RESULTS}

\section{In silico}

Energy minimization of DAAO was carried out. The initial and postminimization potential energies are listed in Table 1.

The conformation and stability of the enzyme were analyzed in terms of its dihedral angles phi and psi using Ramachandran plot. A typical Ramachandran plot consists of a favored, allowed, and disallowed region. The Ramachandran plot of human DAAO is shown in Fig. 1.

Table 2 shows the effect of clozapine $(20 \mathrm{mg} / \mathrm{Kg}$, i.p) on (a) escape latency and (b) time spent, in the Morris water maze test. Clozapine (20 mg/Kg, i.p) was administered to the mice. The mice were then treated with scopolamine $(1 \mathrm{mg} / \mathrm{kg})$ and tested in the Morris water maze test. Probe trial sessions were performed for $60 \mathrm{~s}$. The data represent mean \pm standard error of mean ${ }^{* *} \mathrm{p}<0.001$ compared to scopolamine-treated mice

In vivo

The result of clozapine on scopolamine-induced memory deficit was evaluated using Morris water maze (Fig. 2). Morris water maze evaluates spatial, working, and reference memory. During this study, the escape latency time in animals administered by scopolamine was considerably reduced by clozapine-treated group $\left(20.35 \pm 5.7^{* *}\right)$ compared with the group received vehicle only $(32.21 \pm 2.69)$ as shown in Table 2 .

Table 1: Energy minimization of human DAAO

\begin{tabular}{llll}
\hline S. No & Type of variant & Pre-minimization potential energy & Post-minimization potential energy \\
\hline 1 & HS-DAO & $5150.8530 \mathrm{kcal} / \mathrm{mol}$ & $-744.0501 \mathrm{kcal} / \mathrm{mol}$ \\
\hline *Force field. CHARMM27. Gradient: 0.05 H and LP adjusted. DAAO: D-amino acid oxidase
\end{tabular}

Table 2: Effect of clozapine on scopolamine-induced amnesia

\begin{tabular}{lllll}
\hline S. No & Groups & Treatment & Escape latency (sec) & Time spent in platform quadrant (sec) \\
\hline 1 & Group I & Saline treated $(10 \mathrm{~mL} / \mathrm{kg}$, i.p.) $)($ Vehicle) & $32.21 \pm 2.69$ & $29.37 \pm 3.57$ \\
2 & Group II & Saline $(10 \mathrm{~mL} / \mathrm{kg}$, i.p.,)+Scopolamine $(1 \mathrm{mg} / \mathrm{kg}, \mathrm{i.p})$ & $49.47 \pm 10.32$ & $46.39 \pm 3.8$ \\
3 & Group III & Clozapine $(20 \mathrm{mg} / \mathrm{kg}$, i.p)+Scopolamine $(1 \mathrm{mg} / \mathrm{kg}$, i.p) & $20.35 \pm 5.7^{* *}$ & $22.43 \pm 2.5^{* *}$ \\
\hline
\end{tabular}




\section{DISCUSSION}

To gain better insight for the interactions between antipsychotics and DAAO, molecular docking studies were carried out. The interactions of the ligands with the active site residues (Table 3) of the target are analyzed in terms of the following parameters: Binding energy, number of hydrogen bonds established by the ligand with residues of the active site, $\pi-\pi$ interactions, conformation oriented by the ligand within the active site, and root mean square deviation (RMSD) of the active site residues. The dock score of Autodock is reported in $\mathrm{kcal} / \mathrm{mol}$. Autodock uses the following empirical formula to calculate the free energy of binding:

Binding energy $(\Delta G)=$ Intermolecular energy + van der Waals hydrogen bond desolvation energy + electrostatic energy + total internal energy + torsional energy - unbound energy of the system.

Desolvation energy is a prime parameter that decides a molecules interaction with its pharmacodynamic target [5]. In the biological environment, all drug binding pockets of a target protein remain solvated, and hence, a ligand cannot as such occupy the active site unless it dislodges the water molecules. The similarity of docked structures is measured by computing the RMSD, and clusters are created based on the comparison of conformations and estimated RMSD values [12,13-16]. The docking score of selected ligands with DAO is shown in Table 4, and Fig. 3 shows the 3D docked confirmations of atypical antipsychotic agents.

Impairment of memory and learning and the foremost characteristic manifestation of psychological feature dysfunction are caused by scopolamine, a cholinergic antagonist which interferes with neurotransmitter transmission within the central nervous system [17,18]. The Fig. 2 shows the experimental study, escape latencies in Table 2 it indicates a major decrease for clozapine treated group., whereas the scopolamine-treated group exhibited a characteristic swimming behavior consisting of circling around the pool and longer escape latency indicating impairment of their spatial memory. These results suggested the cognitive enhancement of clozapine.

\section{CONCLUSION}

Clozapine has cognition improvement potential, approving its utilization in alleviating toxic effects of scopolamine. Clozapine being a DAAO inhibitor is an added benefit for the therapy, and it is a better insight for future cognitive studies.

\section{ACKNOWLEDGMENT}

The authors are thankful to the management of School of Pharmaceutical Sciences, Vels Institute of Science Technology and Advanced Studies (VISTAS), for providing excellent research guidance and support. Special thanks to Dr. Samuel Gideon George for the bioinformatics work.

\section{AUTHOR'S CONTRIBUTION}

Both the authors have contributed equally to the research work.

\section{CONFLICTS OF INTEREST}

The authors do not have conflicts of interest.

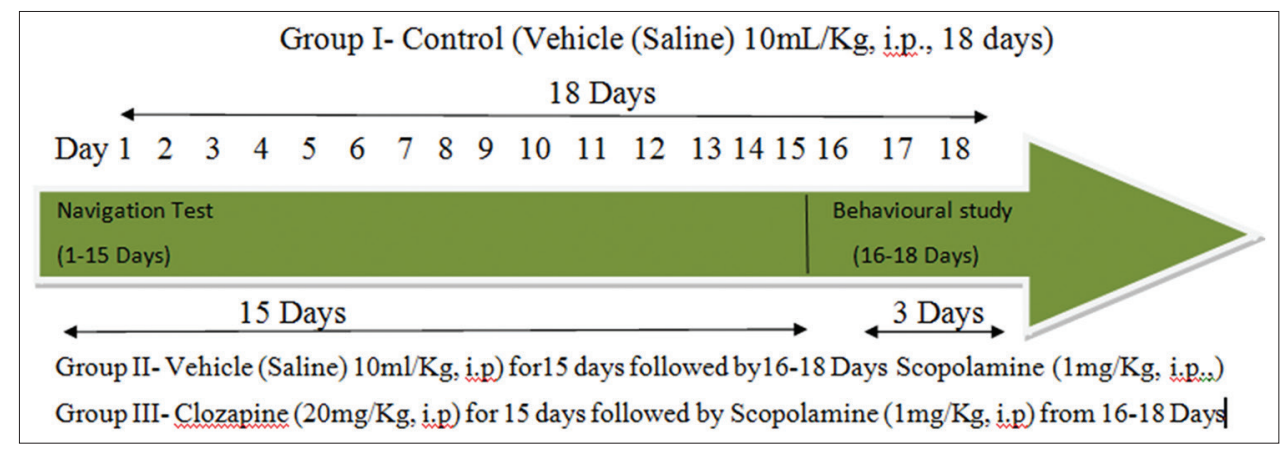

Fig. 2: Scheme of Experimental Schedule: Scopolamine-Induced Amnesia

Table 3: Active site analysis of DAAO

\begin{tabular}{lll}
\hline S. No & Type of variant & Active site residues \\
\hline 1 & DAAO & GLN37 GLN38 LYS39 LYS42 SER43 PR044 LYS45 LEU47 VAL58 PRO59 ARG61 PHE62 ILE75 ALA81 ASP82 TYR86 \\
\hline DAAO: D-amino acid oxidase &
\end{tabular}

Table 4: Molecular docking analysis of antipsychotics with DAAO

\begin{tabular}{llllllll}
\hline Drug & $\Delta \mathbf{G}(\mathbf{k c a l} / \mathbf{m o l})$ & $\mathbf{k I}(\mathbf{m M})$ & $\begin{array}{l}\text { Intermolecular } \\
\text { energy }\end{array}$ & $\mathbf{V H B D E}$ & $\begin{array}{l}\text { Electrostatic } \\
\text { Energy }\end{array}$ & $\begin{array}{l}\text { Total internal } \\
\text { Energy }\end{array}$ & Torsional energy \\
\hline Amisulpride & -3.97 & 1.23 & -6.35 & -6.62 & 0.26 & -1.17 & 2.39 \\
Amoxapine & -4.13 & 0.94 & -7.41 & -7.83 & 0.42 & -0.73 & 3.28 \\
Aripiprazole & -4.25 & 0.76 & -6.34 & -6.53 & 0.19 & -0.23 & 2.09 \\
Blonanserin & -3.67 & 2.05 & -5.75 & -5.9 & 0.15 & -0.01 & 2.09 \\
Brexpiprazole & -5.22 & 0.14 & -7.31 & -7.56 & 0.26 & -0.13 & 2.09 \\
Clocapramine & -2.72 & 10.13 & -6.0 & -6.55 & 0.55 & -0.95 & 3.28 \\
Clorotepine & -3.46 & 2.91 & -5.55 & -5.62 & 0.07 & -047 & 2.09 \\
Clotiapine & -3.04 & 5.88 & -5.13 & -5.76 & 0.63 & -0.49 & 2.09 \\
Clozapine & -2.87 & 7.93 & -4.95 & -5.55 & 0.6 & -0.66 & 2.09 \\
Iloperidone & 1.0 & N/A & -4.67 & -4.85 & 0.18 & 0.09 & 5.67 \\
\hline
\end{tabular}

DAAO: D-amino acid oxidase, VHBDE: Van der Waals hydrogen bond desolvation energy 

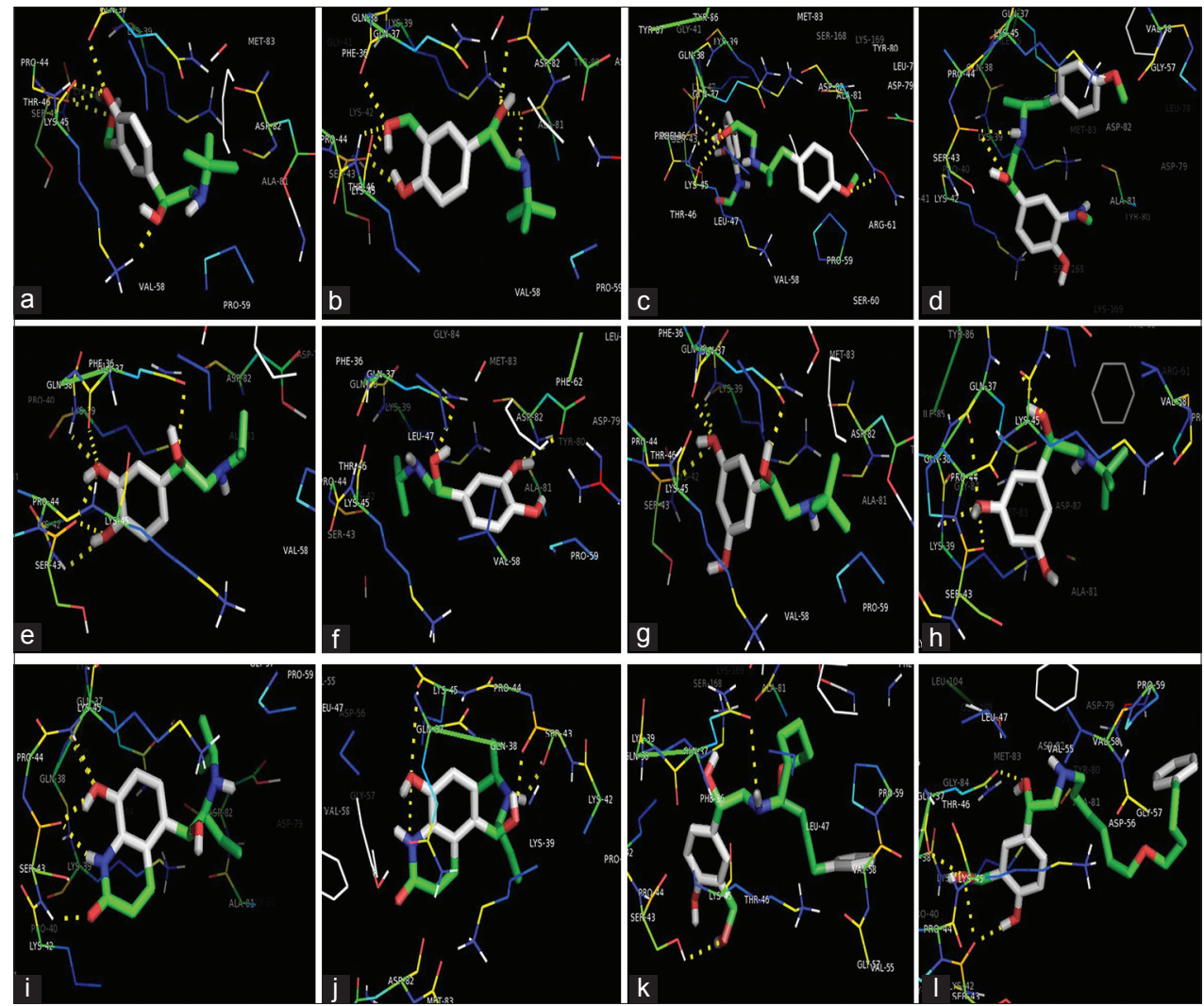

Fig. 3: Three-dimensional docked confirmations of atypical antipsychotic agents. (a) Amisulpride with D-amino acids oxidase (DAA0). (b) Amoxapine with DAAO. (c) Aripiprazole with DAAO. (d) Blonanserin with DAAO. (e) Brexpiprazole with DAAO. (f) Cariprazine with DAAO.

(g) Carpipramine with DAAO. (h) Clocapramine with Arg16 variant. (i) Clorotepine with DAAO. (j) Clotiapine with DAAO. (k) Clozapine with DAAO. (l) Iloperidone with DAAO

\section{REFERENCES}

1. Smith SM, Uslaner JM, Hutson PH. The therapeutic potential of D-amino acid oxidase (DAAO) inhibitors. Open Med Chem J 2010;4:3.

2. Cuesta MJ, Jalón EG, Campos MS, Peralta V. Cognitive effectiveness of olanzapine and risperidone in first-episode psychosis. Br J Psychiatry 2009;194:439-45.

3. Rodefer JS, Nguyen TN, Karlsson JJ, Arnt J. Reversal of subchronic PCP-induced deficits in attentional set shifting in rats by sertindole and a 5-HT6 receptor antagonist: Comparison among antipsychotics. Neuropsychopharmacology 2008;33:2657-66.

4. Lian W, Fang J, Xu L, Zhou W, Kang, Xiong W, et al. DL0410 ameliorates memory and cognitive impairments induced by scopolamine via increasing cholinergic neurotransmission in mice. Molecules 2017;22:pii: E410.

5. Cole JC, Groom CR, Read MG, Giangreco I, McCabe P, Reilly AM, et al. Generation of crystal structures using known crystal structures as analogues. Acta Crystallogr B Struct Sci Cryst Eng Mater 2016;72:530- 41 .

6. Woodley S, Battle P, Gale J, Catlow CA. The prediction of inorganic crystal structures using a genetic algorithm and energy minimisation. Phys Chem Chem Phys 1999;1:2535-42.

7. Laskowski RA, MacArthur MW, Moss DS, Thornton JM. PROCHECK: A program to check the stereochemical quality of protein structures. J Appl Crystallogr 1993;26:283-91.

8. Singh T, Biswas D, Jayaram B. AADS-an automated active site identification, docking, and scoring protocol for protein targets based on physicochemical descriptors. J Chem Inf Model 2011;51:2515-27.

9. Wu G, Robertson DH, Brooks CL, Vieth M. Detailed analysis of gridbased molecular docking: A case study of CDOCKER - A CHARMm- based MD docking algorithm. J Comput Chem 2003;24:1549-62.

10. Acosta JI, Mayer L, Talboom JS, Zay C, Scheldrup M, Castillo J, et al. Premarin improves memory, prevents scopolamine-induced amnesia and increases number of basal forebrain choline acetyltransferase positive cells in middle-aged surgically menopausal rats. Horm Behav 2009:55:454-64.

11. Vorhees CV, Williams MT. Morris water maze: Procedures for assessing spatial and related forms of learning and memory. Nat Protoc 2006;1:848-58.

12. Kim H, Jang C, Yadav DK, Kim MH. The comparison of automated clustering algorithms for resampling representative conformer ensembles with RMSD matrix. J Cheminform 2017;9:21

13. Sindhu T, Rajamanikandan S, Durgapriya D, Anitha JR, Akila S, Gopalakrishnan VK. Molecular docking and qsar studies on plant derived bioactive compounds as potent inhibitors of dekoncoprotein. Asian J Pharm Clin Res 2011;4:67-71.

14. Ganugapati J, Swarna S. Molecular docking studies of antidiabetic activity of cinnamon compounds. Asian J Pharm Clin Res 2014;7:31-4.

15. George SH, Santhlingam K, Chandran ME, Gangwar PA, Gururagavan M. Docking studies of novel coumarin derivatives as arylamine N-acetyltransferance 2 inhibitors. Asian J Pharm Clin Res 2012;5:94-6.

16. Miladiyah IS, Jumina JU, Haryana SM, Mustofa MU. In silico molecular docking of xanthone derivatives as cyclooxygenase-2 inhibitor agents. Int J Pharm Pharm Sci 2017;9:98-104.

17. Lee GY, Lee C, Park GH, Jang JH. Amelioration of scopolamineinduced learning and memory impairment by $\alpha$-pinene in $\mathrm{C} 57 \mathrm{BL} / 6$ mice. Evid Based Complementary Altern Med 2017;2017:4658543.

18. Halicacabum L. In vivo investigation of the neuroprotective potential of cardiospermum. Int J Pharm Pharm Sci 2014;6:64-6. 